\title{
TTR
}

Traduction, terminologie, re?daction

\section{Antoine Berman. L'Épreuve de l'étranger. Culture et traduction dans l'Allemagne romantique. Herder, Goethe, Schlegel, Novalis, Humboldt, Schleiermacher, Hölderlin. Gallimard (coll. TEL), 1995, 311 p.}

\section{Nicole Mallet}

Volume 8, numéro 2, 2e semestre 1995

Technolectes et dictionnaires

URI : https://id.erudit.org/iderudit/037227ar

DOI : https://doi.org/10.7202/037227ar

Aller au sommaire du numéro

Éditeur(s)

Association canadienne de traductologie

ISSN

0835-8443 (imprimé)

1708-2188 (numérique)

Découvrir la revue

Citer ce compte rendu

Mallet, N. (1995). Compte rendu de [Antoine Berman. L'Épreuve de l'étranger. Culture et traduction dans l'Allemagne romantique. Herder, Goethe, Schlegel, Novalis, Humboldt, Schleiermacher, Hölderlin. Gallimard (coll. TEL), 1995, 311 p.] TTR, 8(2), 275-279. https://doi.org/10.7202/037227ar d'utilisation que vous pouvez consulter en ligne. 


\title{
COMPTES RENDUS
}

\author{
Antoine Berman. L'Épreuve de l'étranger. Culture et \\ traduction dans l'Allemagne romantique. Herder, Goethe, \\ Schlegel, Novalis, Humboldt, Schleiermacher, Hölderlin. \\ Gallimard (coll. TEL), 1995, 311 p.
}

La traductologie est une discipline toute neuve dont l'essor au cours des dix dernières années est tout à fait spectaculaire. C'est à peine si naguère on osait énoncer ce vocable sans prendre de précaution. Convaincu que le temps était venu de donner à ce savoir «une forme définie, quasi institutionnelle et établie, propre à permettre son déploiement dans un champ de recherche et d'enseignabilité», Antoine Berman écrivait: «[c]'est ce qu'on a voulu appeler parfois la "traductologie» (d'autres noms moins heureux ont été également avancés)" (L'Épreuve de l'étranger, p. 290). C'était en 1984, dans la première édition de l'ouvrage dont nous accueillons en 1995 la réédition.

Il faut savoir gré aux éditions Gallimard de redonner accès à ce premier ouvrage d'Antoine Berman l'année même où paraît dans leur «Bibliothèque des idées» le livre auquel le regretté critique a travaillé jusqu'à son dernier souffle à l'automne de 1991: Pour une critique des traductions: John Donne, «un maître-livre passionnant et cultivé», pour citer Jean-Michel Desprats qui en rend compte avec admiration et émotion dans TransLittérature (été $1995, \mathrm{n}^{\circ}$ 9). Que de chemin parcouru durant la décennie qui s'achève! On ne relèvera jamais assez la fortune de ce titre programmatique emprunté à Hölderlin et qui contient et détient en raccourci une réflexion sur la pratique et la théorie du traduire. Peut-on rêver, en effet, plus notable consécration que d'être cité en exergue ou en épigraphe par ceux des traductologues, nombreux, sourciers ou ciblistes, qui marchent sur les brisées de celui qui a relancé sur des bases philosophiques et épistémologiques nouvelles le débat pour ou contre la traduction ethnocentrique et l'a installé dans le grand discours moderne de l'altérité?

Il s'agit d'une réédition; par conséquent, c'est pour nous une relecture. Il nous paraît donc singulièrement à propos d'examiner la composition et les grands axes réflexifs de ce texte fondateur sous l'angle de sa postérité. Les répercussions en sont infiniment sensibles, dix ans après, qu'elles émanent de ses disciples ou de ses détracteurs. N'est-ce pas le propre des grands textes que de catalyser le dialogue des contraires? 
L'ouvrage s'ouvre sur un essai fondamental (pp. 11-24), signé par Berman en 1981 et dont la teneur n'a pas vieilli: «La traduction au manifeste», ce dernier terme étant entendu moins dans ses connotations polémiques que dans le sens de la visibilité de la traduction que Berman estime qu'il est nécessaire de substituer à la condition ancillaire (répréhensible) d'une pratique dont il faut repenser les fondements théoriques et l'éthique: «La visée même de la traduction - ouvrir au niveau de l'écrit un certain rapport à l'Autre, féconder le Propre par la médiation de l'Étranger - [...] l'essence de la traduction est d'être ouverture, dialogue, métissage, décentrement» (p. 16). Il est important de bien noter que pour Berman la théorie de la traduction est inséparable de la pratique. Du reste, il préfère au couple théorie/pratique celui plus en accord avec sa démarche de réflexion/expérience. Il le redira dans sa conclusion (p. 300) et le formulera de façon plus ramassée en 1985 dans «La traduction et la lettre ou l'auberge du lointain», où il donne de la «traductologie» la définition suivante: c'est "la réflexion de la traduction sur elle-même à partir de sa nature d'expérience» (Les Tours de Babel, Trans-Europ-repress, Mauvezin, p. 39). Une réflexion fructueuse sur la traduction et les traducteurs doit selon lui s'articuler autour de trois axes: une Histoire de la traduction, une Éthique de la traduction, une Analytique de la traduction. Gallimard annonce la parution posthume de deux ouvrages de Berman, en chantier au moment de sa mort, et qui attestent du travail qu'il accomplissait dans ce sens: Traduire Benjamin et Jacques Amyot traducteur français ${ }^{1}$. Berman considère que «[l]a constitution d'une histoire de la traduction est la première tâche d'une théorie moderne de la traduction" (p. 12). Le grand projet d'une Histoire thématique de la traduction dont nos collègues canadiens, Jean Delisle et Judith Woodsworth, sont les maitres d'œuvre se situe à coup sûr dans le droit fil de ce vœu.

Conformément aux promesses du sous-titre de son ouvrage (Culture et traductiondans l'Allemagneromantique), Berman pose lui-même la première pierre de cet édifice qui doit abriter un discours diversifié, fécond et ouvert, entreprise qu'il décrit dans sa conclusion comme «une archéologie de la traduction européenne») (p. 280). Pour ce faire, il se tourne vers une phase-clé de cette archéologie, une époque qu'en tant que philosophe et traducteur il connaît dans le détail; une période durant laquelle les problèmes inhérents au rapport avec l'étranger se sont posés avec une acuité particulière et qu'il va reconstruire: celle des Romantiques d'Iéna regroupés autour de la revue Athenäum fondée par le frères Schlegel. L'analyse des travaux de Friedrich et August Wilhelm Schlegel ainsi que de ceux de Novalis qui ont contribué à la

1. L'éminent érudit avait donné en primeur un extrait de cet ouvrage pour le tout premier numéro de $T T R$, en 1988: «De la translation à la traduction», pp. 23-40. 
"révolution romantique» couvre les cinq chapitres qui occupent le cœur du livre (ch. 5-9, pp. 111-225). Berman ne craint pas de parler de révolution à propos de la pensée romantique «ivre de pathos critique» (p. 113). Il analyse d'abord le concept de «versabilité infinie» qui joue un rôle primordial dans l'encyclopédisme de Novalis; l'émergence de plus en plus prononcée de celui de germanité; la théorie du langage poétique qui s'appuie sur la distinction entre langage de nature et langage d'art; le télescopage entre l'acte de traduire et celui de critiquer propre à la spéculation idéaliste des Romantiques d'Iéna et qui débouche sur la conception monologique d'une «traduction mythique» (se reporter en particulier aux pages 177-204); la volonté enfin de tout traduire de Wilhelm Schlegel que Berman voit comme un «omnitraducteur» plutôt que comme le talentueux polytraducteur qu'il fut.

De part et d'autre de cette étude centrale, Berman a placé des chapitres qui élargissent le débat et s'inscrivent dans ses marges, soit en le fondant, soit en le suivant jusque dans ses prolongements les plus modernes et les plus paradoxaux. En amont de ce noyau spéculatif, Berman inscrit un chapitre sur «Luther ou la traduction comme fondation» (ch. 1, pp. 43-60). Il discute les paradoxes du concept historico-culturel de la Verdeutschungcher au traducteur allemand de la Bible que les théoriciens de la fin du XVIII ${ }^{\circ}$ perdront de vue jusqu'à ce que Hölderlin le reprenne avec son idée d'un triple «apprentissage du propre (la patrie, le natal, le national)» (p. 258), inaugurant par là «une nouvelle époque de la poésie et de la traduction en Allemagne» ( $p$. 225). Le chapitre sur Luther est suivi de trois autres consacrés à retracer l'importance des idées de Herder et surtout de Goethe (éparses dans son cuvre, particulièrement explicites dans le Divan occidental-oriental)dans l'édification de la philosophie romantique de la culture, de l'histoire et du langage. Berman y sonde en particulier le concept de la Bildung et ses différents registres et celui de Weltliteratur et les impératifs que ceux-ci entraînent vis-à-vis de la traduction.

En aval de ce corpus centré sur le groupe de l'Athenäum, Berman examine les écrits sur la traduction de W. von Humboldt et de F. Schleiermacher. Rappelons qu'il est l'auteur de la première traduction française de la conférence prononcée par Schleiermacher à l'Académie Royale des Sciences de Berlin en 1813, Über die verschiedenenMethoden des Übersetzens (Des différentesméthodes du traduire, publiée en présentation bilingue dans Les Tours de Babel, pp. 279-347). L'herméneuticien français salue Schleiermacher comme «le fondateur de cette herméneutique moderne qui se veut une théorie de la compréhension» (p. 227). Ce n'est guère l'avis de Lawrence Venuti, grand pourfendeur pourtant de la traduction ethnocentrique. Dans un article récent («Genealogies of Translation theory: Schleiermacher», TTR, vol. IV, $\mathrm{n}^{\circ} 2$, pp. 125-150), Venuti rend certes à Berman le respect idéologique qui lui est dû, 
mais il reprend le débat sur des bases nettement politiques et dénonce la vocation hégémonique dévolue au traducteur: «Schleiermacher is enlisting his privileged translation method in a cultural political agenda, wherein an educated elite controls the formation of a national culture by refining its language through foreignizing translations») (p. 131).

Plus virulente est la diatribe de Douglas Robinson dans «Foreignizing experience» (Canadian Review of Comparative Literature/RevueCanadienne de Littérature Comparée, sept.-déc. 1993, pp. 417-433). L'article est écrit à l'occasion de la traduction anglaise de L'Épreuve de l'étranger due à Stefan Heyvaert (SUNY Press, 1992) dont la langue maternelle est le néerlandais: épreuve de l'étranger au deuxième degré pour l'ouvrage de Berman; le cas de figure ne manque pas de saveur! Avant de descendre en flèche les choix stylistiques du traducteur au nom d'un purisme de la langue qui réprouve toute entreprise littéraliste, Robinson taxe le mysticisme de Berman de «protofasciste» et reproche au traductologue de fétichiser la pureté de la langue en assimilant les concepts de pureté et d'altérité. Berman n'est plus là pour se justifier mais il est permis de se demander si son option poétologique aurait apprécié une telle radicalisation de ses idées. Après tout, une lecture en appelle une autre; l'Autre n'est jamais le même pour un autre; c'est de bonne guerre et c'est là justement l'une des grandes leçons éthiques du livre de Berman.

Après cette digression dans l'espace combatif et partial de la polémique, revenons à l'ample balayage historique, profond et subtil, de Berman. Le livre s'achève sur Hölderlin à qui le génie de traducteur à l'œuvre a insufflé la notion de traduction dialogique qui, même motivée historiquement, le situe radicalement en avance sur son temps. Berman salue son audace à définir «l'acte de traduire dans la déroutante multiplicité de ses registres» (p. 277). En suivant à la fois de façon synchronique et diachronique ce discours des écrivains allemands du XIX ${ }^{e}$ siècle, Berman révèle comment et pourquoi la réflexion sur la traduction pénètre dans l'horizon de l'herméneutique et des sciences du langage.

Loin d'être une clôture, la conclusion s'ouvre sur un faisceau de perspectives résolument modernes qu'on pourrait rassembler ainsi: la traduction doit être à la fois objet et sujet de savoir; la pratique du traduire se doit d'être revalorisée, et il importe de réintroduire dans la spéculation sur le traduire la notion et la valeur du sujet traduisant ainsi que de nuancer les stratégies de la transparence. Berman affirme la nécessité historique et éthique des retraductions et appelle de ses vœux une orientation interdisciplinaire des recherches théoriques sur la traduction. À des degrés divers, les travaux des traductologues du monde occidental répondent à l'un ou à plusieurs de ces appels. Les enquêtes des sémioticiens de l'école polysystémique de Tel Aviv et celles socio-critiques 
de leurs disciples belges et canadiens peuvent se réclamer de cette obédience pluri/interdisciplinaire et manipuler à l'infini le concept d'altérité: «Regardons comment et à quelles conditions le «discours» porté par les textes étrangers peut venir s'insérer dans le «discours» de la société qui le reçoit», propose Annie Brisset dans Sociocritiquede la traduction. Théâtre et altéritéau Québec (19681988) (Longueuil, Le Préambule, 1990, p. 25). Cette étude magistrale, dans sa démarche et ses aboutissements, est une belle preuve de l'ouverture de l'approche bermanienne. Antoine Berman a d'ailleurs signé la préface du livre. Certaines publications témoignent de la fécondité inépuisable de cette notion de l'épreuve de l'étranger. Le groupe Palimpsestes de la Sorbonne lui a consacré une de ses sessions ( $L$ 'étranger dans la langue, $\mathrm{n}^{\circ} 6,1991$ ). Dans son tout dernier livre, Le Trafic des langues (Les Éditions du Boréal, 1994), Sherry Simon pourchasse cette idée jusque dans ses plus paradoxaux retranchements (voir en particulier son septième et dernier chapitre: «Traduire l'étranger en nous»). Signalons encore le volume collectif sous la direction de Lawrence Venuti: Rethinking Translation (Routledge, 1992) qui prend en compte les théories modernes de la réception, les problèmes du langage et de la subjectivité pour repenser la notion de l'altérité en traduction dans un contexte philosophique et politique.

Cet ouvrage, porteur d'une pensée riche, dense et généreuse est un exemple de rigueur spéculative, d'intelligence critique, d'ouverture à cet Autre qu'est le lecteur/la lectrice. Il faut relire et repenser ce livre inspiré, d'une érudition de bon aloi dont la modestie dans la quête du vrai ne cède jamais le pas à la cuistrerie et qui garde toute sa vigueur et sa pertinence. C'est une somme critique qui s'avérera fort utile aux chercheurs, germanistes, comparatistes qui cuuvrent à débusquer les filiations et les dérives des théories du texte dans l'Europe occidentale. Pour ceux et celles qui ne lisent pas l'allemand, et fonctionnent en français, ce volume, par l'abondance et la longueur des extraits traduits, constitue une précieuse anthologie des écrits sur la question. Avec son texte-frère des Tours de Babel, L'Épreuve de l'étranger demeure pour le monde de la traductologie l'un des grands textes novateurs de notre temps dans le champ d'investigation de la problématique actuelle du traduire.

Nicole MALLET Université d'Alberta 